\title{
Propagation of Free Electrons Carrying Orbital Angular Momentum Through Magnetic Lenses
}

\author{
David Shook, Benjamin J. McMorran \\ Department of Physics/University of Oregon, Eugene, OR, USA
}

Free electrons with orbital angular momentum (OAM) in quantized states can be produced inside of a TEM using nanofabricated holographic diffraction gratings. The images of these electron vortex beams show ring-shaped projections of their helical wavefronts. These wavefronts are tilted both azimuthally and radially by a degree dependent on the OAM. As a model for these beams, we can consider a coherent superposition of classical straight line trajectories, each of which are skewed from the optical axis.[1] This semi-classical model of an electron vortex beam was simulated using COMSOL Multiphysics in order to better understand what effect topological charge (i.e. skew angle) has on beam waist position, size and shape after propagating through various electron optics. Specifically, the effects of a magnetic lens and a magnetic quadrupole lens acting on an electron vortex beam were investigated.

The magnetic lens was modeled as a current coil with approximately a 1 Tesla magnetic field at its center. We simulate an electron vortex beam emerging from an aperture $15 \mathrm{~mm}$ in front of the lens, by preparing an initial bundle of $300 \mathrm{keV}$ electrons with azimuthally skewed straight line trajectories. These non-axial electrons spiral about the optical axis as a result of the Lorentz force (Figure 1A). The average radius of the resulting vortex beam as it propagated through the magnetic field was determined from the simulated trajectories and the focus behind the lens located (Figure 1, B and C). These simulations indicate that there is a displacement in the location of the new beam waist that depends on OAM. This suggests a beam defocus for electron vortex beams.

In an electron microscope, a stigmator is used to control symmetry of the beam. These elements are tunable magnetic quadrupoles which serve as the electron optical equivalent of cylindrical lenses. Experiments with electron vortex beams show that the shape and orientation of a stigmated beam depends upon the magnitude and sign of the OAM (Figure 2, A and B). To simulate this, a quadrupole lens was modelled as four permanent magnets with the poles reversed on two of the magnets. Again, a circular bundle of $300 \mathrm{keV}$ electrons with skewed initial trajectories were allowed to propagate through the resulting field. A planar view of the bundle of beams after passing through the quadrupole lens agrees with TEM images of stigmated electron vortex beams (Figure 2C). The image is seen to be clearly tilted in the opposite direction for opposite OAM of the beam.

In an effort to interpret TEM images collected using electron vortices, we will continue to use this semi-classical model of electron vortex beams to investigate the combined effects of OAM and beam tilt, rotation center, and lens aberrations.

[1] B. J. McMorran et al, Science 331 (2011), 192-195.

[2] The authors acknowledge funding from U.S. Department of Energy, Office of Science, under the Early Career Research Program. 

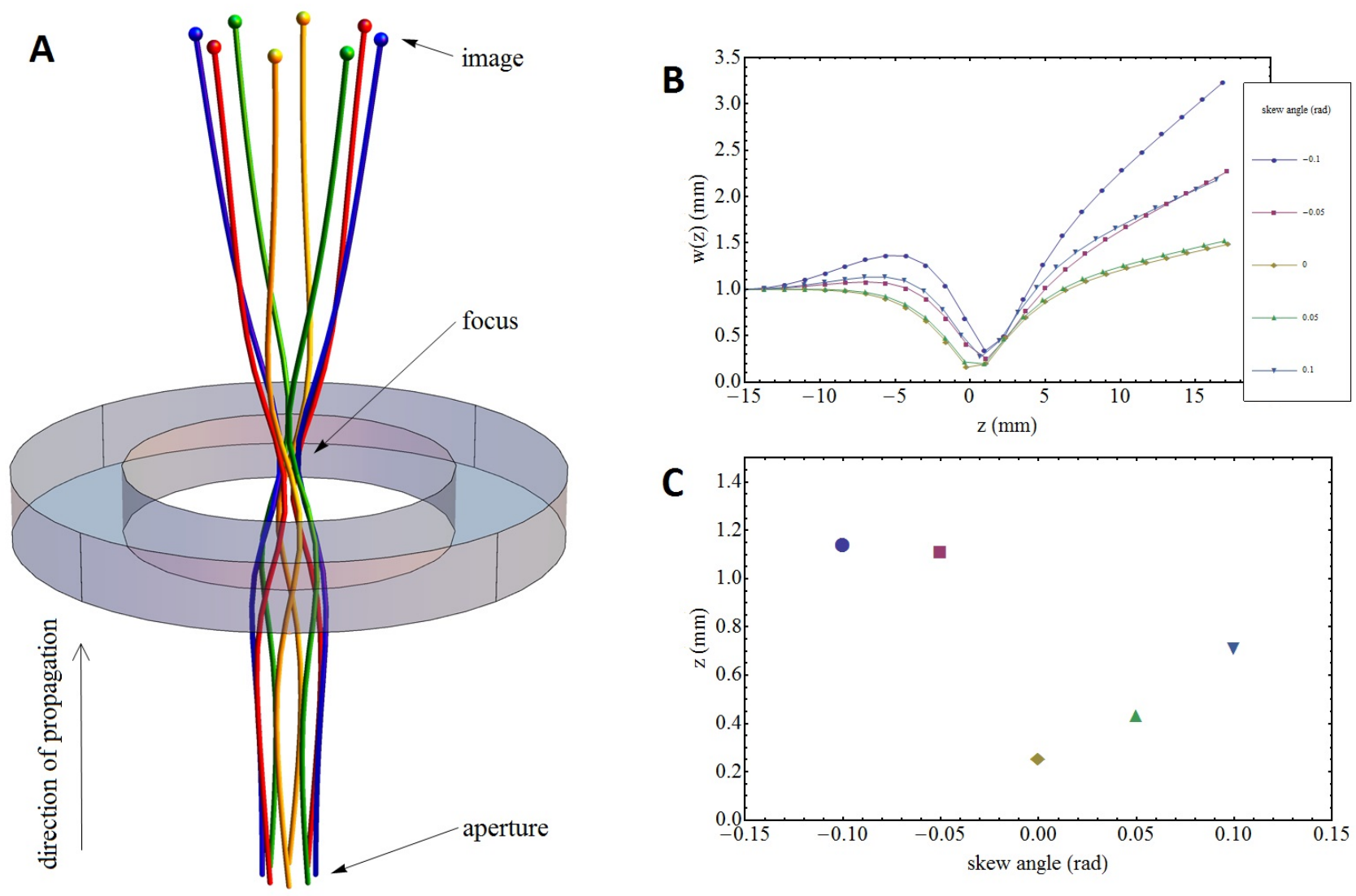

Figure 1. (A) Trajectories of the simulated electron vortex beam. (B) Average beam width and (C) focus of simulated electron vortex beams with different OAM passing through a magnetic lens.
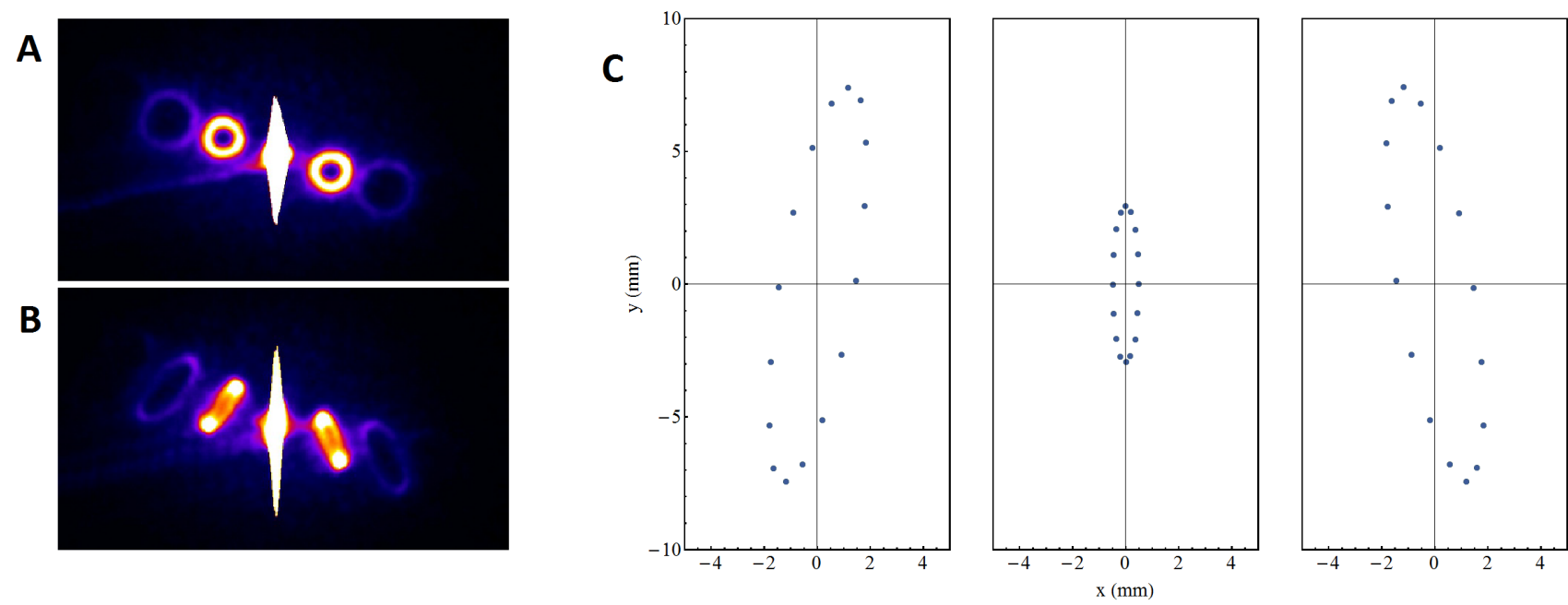

Figure 2. (A) Non-stigmated and (B) stigmated images of multiple vortex beams. (C) Simulations of vortex beams with OAM equal in magnitude but opposite in charge of each other (left and right) and without OAM (center). 